\title{
A CLOSED FORM SOLUTION FOR NON-LINEAR DEFLECTION OF NON-STRAIGHT LUDWICK TYPE BEAMS USING LIE SYMMETRY GROUPS
}

\author{
M.Amin Changizi ${ }^{1}$, Davut Erdem Sahin ${ }^{2}$, Ion Stiharu ${ }^{3}$ \\ ${ }^{1}$ Knowledge Engineering, Intelliquip Co. \\ 3 W Broad St, Bethlehem, PA 18018, USA \\ 2 Bozok University, Department of Mechanical Engineering 66200, Yozgat, Turkey \\ ${ }^{3}$ Concordia University, Department of Mechanical and Industrial Engineering, \\ 1455 De Maisonneuve Blvd. W., Montreal, Quebec, Canada, H3G 1 M8 \\ achangizi@intelliquip.com, davut.sahin@bozok.edu.tr, istih@encs.concordia.ca
}

\begin{abstract}
Micro Electro Mechanical Systems (MEMS) have found a large range of applications over the recent years. One of the prodigious application of micro-cantilever beams that is in use is represented by AFM probes (Atomic Force Microscopy). The AFM principle is based on the realtime measurement of the deflection of a micro-beam while following a surface profile. Hence, the prior knowledge of the deflection of beams has been of great interest to designers. Although both analytical and numerical solutions have been found for specific type of loads, there is no general solution specifically formulated for micro-cantilever beams that are not geometrically perfectly straight. Hence, the problem has not been specifically considered so far. The current work presents an analytical method based on Lie symmetry groups. The presented method produces an exact analytical solution for the deflection of Ludwick type beams subjected to any point load for nonstraight beams. The Lie symmetry method is used to reduce the order of the Ordinary Differential Equation (ODE) and formulate an analytical solution of the deflection function. The result is compared with an analytical solution for a particular case that is available in the open literature. It was found that the two results coincide.
\end{abstract}

Keywords: Non-linear deflection, Ludwick material, Lie group symmetry, micro-cantilever beams

\section{Introduction}

The nonlinear deflection of beams loaded by various loads and subjected to different boundary conditions has been largely investigated [1-5]. The prediction of the deflection of beams has been of great interest to researchers and designers [1-5, 8-11, 19]. This seems to be a mundane problem as it is the subject of many textbooks on elementary mechanics of materials. However, the general problem for beams that are not geometrically straight has not been investigated so far in a systematic fashion. Moreover, materials used in applications such as microstructures exhibit deflections that are within the non-linear domain. This is one of the reasons that the Ludwick materials concept resurfaced.

Materials which obey Ludwick constitutive law [1] of stress-strain are called Ludwick materials. This aspect will be further discussed further in this paper in section 3. The evidence shows that micro and nano-technologies will significantly grow in the near future. This trend motivates this research. The fabrication techniques used in micro and nan0 fabrication may yield imperfect geometries or slightly deformed components at times. Hence, the investigation of non-straight structures represents a necessary investigation to understand the performances of slightly imperfect microstructures.

This research presents an approach in solving both geometrical and material nonlinear behaviour of cantilever beams based on Lie symmetry. A general analytical solution of the problem is presented below. Hence, the objective of the present work is to investigate a versatile mathematical method that leads to a solution of the deflection of geometrically non-straight micro-cantilever beams made from Ludwick type materials and subjected to point force. The Lie symmetry method will be used for finding an analytical solution for the nonlinear ODE's. Lie symmetry is applied to the nonlinear ODE which has been formulated for large deflection of micro-cantilever beams built from a nonlinear material of Ludwick type. This ODE cannot be used for conditions for which there is no residual stress in the unloaded beam. A PDE is used to model this case.

The solution of the differential equation for the large deflection cantilever beams loaded by point forces at the tip was derived in 1945 [2]. In that 
approach the differential equation describing the slope of the beam versus the length of the deflected curve was formulated and solved by complete second and first kind elliptic integrals. A numerical solution was provided [3] for the differential equation of the slope versus length of the deflected beam by considering the shear force. In this work solutions were derived from finite differences method for the ODE describing a cantilever and a simple supported beam subjected to uniform distributed forces. For the simple supported beams under point forces the same method was used to solve the constitutive ODE. A numerical solution for the tapered cantilever beam under a point force at the tip was presented in 1968 [4]. A nondimensional ODE was solved by computer at that time. The performance of a cantilever beam made from materials exhibiting nonlinear properties subjected to a point force was studied in [5].

In the results of the research presented in [6] the deflection equation was derived for a Ludwick experimental strain-stress curve. The integral equation was solved numerically to calculate the deflection and the rotation at the tip of the beam. The work in [7] used power series and neural network to solve for the large deflection of a cantilever beam under a point force at the tip. The constitutive equation that expresses the curvature of the beam made from a non-linear material is a nonlinear ODE. The authors of [8] studied large deflections of cantilever beams made from nonlinear elastic materials subjected to uniform distributed forces and a point force at the tip. They developed a system of nonlinear ODEs to model the system which was further solved by the Runge-Kutta method. The authors in [9] used the same method as in [2] to solve the large deflection equation of a cantilever under a point force at the tip. In their work they validated the theoretical work using experimental results. The non-dimensional formulation was used to simplify the nonlinear deflection and reduce the problem to linear analysis. They showed that the nonlinear assumption of small deflection yields the same results as those found through the linear analysis. Two dimensional loads on cantilever beam with point force loading at the free end was studied for both non-prismatic and prismatic beams [10]. A model for the general loading conditions on beams was formulated as a nonlinear Partial Differential Equations (PDE) [10]. Further, a numerical solution based on a non-dimensional equation was presented [10]. A theoretical study on the large deflection of a cantilever beam subjected to a tip moment made form a nonlinear bimorph material was presented using a numerical solution in [11]. The authors used an exact formulation for the deflection of a cantilever with a moment applied at the tip. The authors used a system of ODEs to numerically solve the large deflection of the cantilever beam under a uniform distributed and tip point load.
They presented a comparison between numerical and experimental results. The large deflection of a non-prismatic cantilever beam subjected to different type of continuous and discontinuous loading was studied by finite difference methods [13]. The authors formulated the problem based on [10] and further used quasi-linearization central finite differences method to solve the problem. The large deflection of a cantilever beam subjected to a point load at the tip was studied by a homotopic analysis method (HAM) and an explicit solution for it was presented in [13]. Large deflection of a non-uniform spring-hinged cantilever beam under a follower point force at the tip was formulated and numerically solved [15]. Large deflection of curved beams under a tip load was studied analytically by the Lie symmetry method in [16].

In the present work the Lie group symmetry approach is used as a feasible alternative to express the curvature of a not straight beam deflecting beyond the linear level and made from a non-linear material for which stress-strain correlation follows the Ludwick formulation. Lie group symmetry is a versatile mathematical method that has recently been extended to solve some engineering problems.

\section{Nomenclature}

$\begin{array}{cl}\sigma & \text { Stress } \\ \text { E } & \text { Young's Modules } \\ \varepsilon & \text { Strain } \\ \mathrm{n} & \text { Power coefficient } \\ \mathrm{A} & \text { Area of cross section } \\ \mathrm{I} & \text { Moment of inertia } \\ \mathrm{h} & \text { Thickness of beam } \\ \mathrm{V} & \text { Vertical component of force } \\ \mathrm{H} & \text { Horizontal component of force } \\ \mathrm{P} & \text { Point force at tip of beam } \\ \theta & \text { Angle of point force } \\ \varphi & \text { Angle of Curvature } \\ \rho & \text { Curvature of beam } \\ \delta_{\mathrm{h}} & \text { Horizontal deflection of tip } \\ \delta_{\mathrm{v}} & \text { Vertical deflection of tip } \\ \mathrm{y} & \text { Distance from neutral axis of beam } \\ \xi, \eta & \text { Components of infinitesimal } \\ & \text { transformation } \\ \mathrm{U} & \text { Transformation operation } \\ \alpha & \text { The parameter of the group }\end{array}$

\section{Ludwick Materials}

The relation between stress and strain for linear materials is based on Hooke's Law which is a linear formulation [19]. A large range of nonlinear materials which exhibit nonlinear stress-strain behaviour can be defined by the Ludwick relation [5]. The Ludwick stress-strain relation is defined as following: 


$$
\sigma=\mathrm{E} \times \varepsilon^{\frac{1}{\mathrm{n}}}
$$

Here, E and n represent constants related to material properties. The behaviour of this material for different $n$ is illustrated in figure 1 [5].

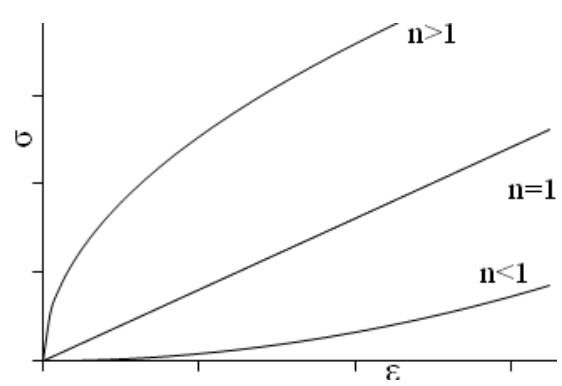

Figure 1: Ludwick material stress-strain behaviour

In the current work the large deflection of microcantilever beams made from Ludwick type materials is investigated. For an example, in AFMs, certain applications require large deflection of beams which may be non-straight due to the variability of micromachining processes. This calls for a model based on Ludwick type materials as the deflection of such elements may exceed many times the initial thickness of the structure. For this case the moment of area of a rectangular cross section of the-Ludwick type material needs to be defined. In the open literature has been shown that the moment of inertia can be expressed as in (3.2) [17]:

$$
I_{n}=\int y^{\frac{n+1}{n}} d A=\left(\frac{1}{2}\right)^{\frac{n+1}{n}}\left(\frac{n}{2 n+1}\right) b h^{\frac{2 n+1}{n}}
$$

\section{Formulation of the Large Deflection of the Not-Straight Beam Problem}

For a curved AFM beam, a point force at the tip can be considered as illustrated in figure 2 [16]. The moment at any cross section of the beam can be written as:

$\mathrm{M}(\mathrm{x}, \mathrm{y})=\mathrm{V} \times \mathrm{x}+\mathrm{H} \times \mathrm{y}+\mathrm{M}_{0}$

where:

$$
\begin{aligned}
& V=P \sin (\theta) \\
& H=P \cos (\theta) \\
& M_{0}=P \cdot u
\end{aligned}
$$

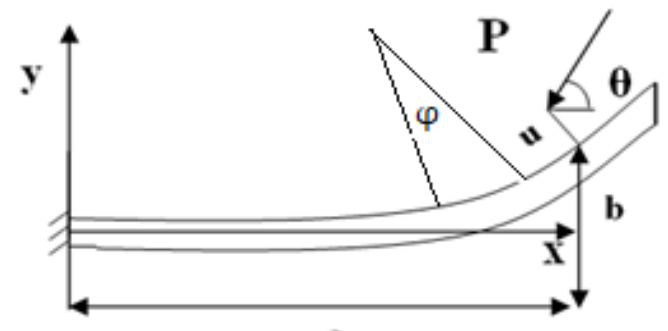

a
Figure 2: A non-straight AFM micro-cantilever beam under tip load and moment.

Here $\mathrm{u}$ represents the distance from the tip to neutral axis of the cantilever beam. Euler-Bernoulli moment-curvature relationship results in [5]:

$$
\frac{d \varphi}{d \rho}=\frac{M(x, y)}{E\left(\frac{1}{2}\right)^{\frac{n+1}{n}}\left(\frac{n}{2 n+1}\right) b h^{\frac{2 n+1}{n}}}
$$

Where:

$$
\begin{aligned}
& \frac{\mathrm{dx}}{\mathrm{d} \rho}=\cos (\varphi) \\
& \frac{\mathrm{dy}}{\mathrm{d} \rho}=\sin (\varphi)
\end{aligned}
$$

Differentiation of equation (4.2) yields:

$$
\frac{\mathrm{d}^{2} \varphi}{\mathrm{d} \rho^{2}}=-\frac{\mathrm{V} \cos (\varphi)+\mathrm{H} \sin (\varphi)}{\mathrm{E}\left(\frac{1}{2}\right)^{\frac{\mathrm{n}+1}{\mathrm{n}}}\left(\frac{\mathrm{n}}{2 \mathrm{n}+1}\right) \mathrm{bh} \frac{\frac{2 \mathrm{n}+1}{\mathrm{n}}}{}}
$$

The boundary conditions can be defined as following:

$\varphi \|_{p=0}=0$

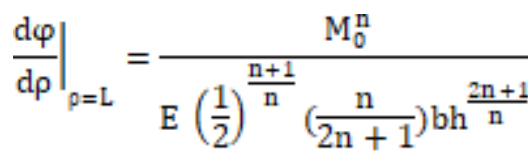

where $L$ is the length of cantilever. To solve (4.5) one should use a mathematical groups which are considered in this paper are point symmetric transformations [19] which means that each point $(\mathrm{x}, \mathrm{y})$ on the curve move into $(\mathrm{x} 1, \mathrm{y} 1)$ :

$\mathrm{x}_{1}=\phi\left(\mathrm{x}_{v} \mathrm{y}, \alpha\right) \quad \mathrm{y}_{1}=\psi\left(\mathrm{x}_{v} \mathrm{y}_{0} \alpha\right)$

Where $\Phi$ and $\Psi$ are diffeomorphism ( $C$ ). If any transformation preserves the shape of a curve and it maps the curve on itself, the transformation is called symmetry [19]. The transformations (4.8) which satisfies the group properties is called oneparameter group [19] and $\alpha$ is called the parameter of the group. For a one-parameter group the infinitesimal transformation is defined as [19]:

$$
\begin{aligned}
& \mathrm{Uf}=\xi(\mathrm{x}, \mathrm{y}) \frac{\partial \mathrm{f}}{\partial \mathrm{x}}+\eta(\mathrm{x}, \mathrm{y}) \frac{\partial \mathrm{f}}{\partial \mathrm{y}} \\
& \xi(\mathrm{x}, \mathrm{y})=\left.\frac{\partial \Phi}{\partial \alpha}\right|_{\alpha=0} \eta(\mathrm{x}, \mathrm{y})=\left.\frac{\partial \psi}{\partial \alpha}\right|_{\alpha=0}
\end{aligned}
$$

One can show that for a second order ODE infinitesimal transformation, $\eta^{[2])}$ can be calculated as in $[13,14]$ :

$$
\begin{gathered}
\eta_{\rho \rho}+\left(2 \eta_{\rho \varphi}-\xi_{\rho \rho}\right) \dot{\varphi}+\left(\eta_{\varphi \varphi}-2 \xi_{\rho \varphi}\right) \dot{\varphi}^{2}-\xi_{\varphi \varphi} \dot{\varphi}^{3}+\left(\eta_{\varphi}-2 \xi_{\rho}-3 \xi_{\varphi} \dot{\varphi}\right) \omega \\
=\xi \omega_{\rho}+\eta \omega_{\varphi}+\left(\eta_{\rho}+\left(\eta_{\varphi}-\xi_{\rho}\right) \dot{\varphi}-\xi_{\varphi} \dot{\varphi}^{2}\right) \omega_{\dot{\varphi}}
\end{gathered}
$$

where $\omega$ is right hand side of (4.5): 
$\omega=-\frac{V \cos (\varphi)+H \sin (\varphi)}{E\left(\frac{1}{2}\right)^{\frac{n+1}{n}}\left(\frac{n}{2 n+1}\right) b h^{\frac{2 n+1}{n}}}$

$\mathscr{\varphi}=\frac{\mathrm{d} \varphi}{\mathrm{d} \rho}$

Solving equation (4.11) as a system of PDEs, one can calculate $\xi$ and $\eta$. Rotation, translation and scaling as

a Lie symmetry of equation (4.5) could be found with the following transformations:

$\xi=C_{1}+C_{2} P+C_{3} \varphi$

$\eta=\mathrm{C}_{4}+\mathrm{C}_{5} \rho+\mathrm{C}_{6} \varphi$

where:

$\mathrm{C}_{1}, \mathrm{C}_{2}, \mathrm{C}_{3}, \mathrm{C}_{4}, \mathrm{C}_{5}, \mathrm{C}_{6}$ are constants of transformation.

Substituting equation (4.12) into equation (4.11) yields:

$$
\left(C_{6}-2 C_{2}-3 C_{2} \dot{\varphi}\right)\left(-\frac{V \cos (\varphi)+H \sin (\varphi)}{E\left(\frac{1}{2}\right)^{\frac{n+1}{n}}\left(\frac{n}{2 n+1}\right) b h^{\frac{2 n+1}{n}}}\right)=\left(\frac{V \cos (\varphi)-H \sin (\theta)}{E\left(\frac{1}{2}\right)^{\frac{n+1}{n}}\left(\frac{n}{2 n+1}\right) b h^{\frac{2 n+1}{n}}}\right)\left(C_{4}-C_{2} \rho+C_{6} \dot{\varphi}\right)
$$

Decomposing equation (4.13) by compare ing terms will yield:

$\mathrm{C}_{2}=\mathrm{C}_{\mathrm{a}}=\mathrm{C}_{4}=\mathrm{C}_{5}=\mathrm{C}_{6}=0$

Hence:

$\xi=\mathrm{C}_{1}$

$\eta=0$

One can refer to appendix B in [19] for detail calculation of constants for $\xi$ and $\eta$. Equations (4.15) provide a general transformation for equation (4.5) based on a general transformation like (4.12). (4.15) presented a linear transforming along $\xi$-axes.

One can consider $\xi$ and $\eta$ as follows:

$$
\begin{aligned}
& \xi(\rho, \varphi)=1 \\
& \eta(\rho, \varphi)=0
\end{aligned}
$$

The point symmetry operator (4.9) when using (4.16) and (4.17) will be simplified as:

$\mathrm{X}=\frac{\partial}{\partial \rho}$

In the next step the attempt to solve the ODE given by equation (3.5) requires introduction of two variables which are called canonical coordinates, $\mathrm{r}(\rho, \varphi)$ and $s(\rho, \varphi)$, which will be defined below. The coordinate $\mathrm{r}(\rho, \varphi)$ is the solution of the following ODE:

$\frac{\mathrm{d} \varphi}{\mathrm{d} \rho}=\frac{\eta(\rho, \varphi)}{\xi(\rho, \varphi)}$

and $s(\rho, \varphi)$ will be:

$\left.s(\rho, \varphi)=\int \frac{d \rho}{\xi\left(\rho, \varphi\left(r_{v}, \rho\right)\right)}\right)\left.\right|_{r=r(\rho, \varphi)}$

Substituting equations (4.16) and (4.17) into equation (4.19) and (4.20), respectively will yield:

$\mathbb{r}(\rho, \varphi)=\varphi$

$s(\rho, \varphi)=\rho$
A new variable, $u(r) \times$ can be defined as:

$\mathrm{u}(\mathrm{r})=\frac{1}{\frac{\mathrm{d} \varphi}{\mathrm{d} \rho}}$

It can be shown that (for detail calculation based on Contact method, please see appendix C in [19]):

$\frac{\mathrm{du}(\mathrm{r})}{\mathrm{dr}}=-\frac{\frac{\mathrm{d}^{2} \varphi}{\mathrm{d} \rho^{2}}}{\left(\frac{\mathrm{d} \varphi}{\mathrm{d} \rho}\right)^{2}}$

Hence,

$\frac{d^{2} \varphi}{d \rho^{2}}=-u(r)^{-3} \frac{d u(r)}{d r}$

by replacing equations (4.23) and (4.25) into equation-(4.5) gives:

$\frac{\mathrm{du}(\mathrm{r})}{\mathrm{dr}}=\left(\frac{\mathrm{Vcos}(\varphi)+H \sin (\varphi)}{\mathrm{E}\left(\frac{1}{2}\right)^{\frac{\mathrm{n}+1}{\mathrm{n}}}\left(\frac{\mathrm{n}}{2 \mathrm{n}+1}\right) \mathrm{bh} \mathrm{h}^{\frac{2 \mathrm{n}+1}{\mathrm{n}}}}\right) \mathrm{u}(\mathrm{r})^{\mathrm{a}}$

Substitution of equations (4.21) and (4.22) into equation (4.26) will yield:

$\frac{\mathrm{du}(\varphi)}{\mathrm{d} \varphi}=\left(\frac{\mathrm{V} \cos (\varphi)+\mathrm{H} \sin (\varphi)}{\mathrm{E}\left(\frac{1}{2}\right)^{\frac{\mathrm{n}+1}{\mathrm{n}}}\left(\frac{\mathrm{n}}{2 \mathrm{n}+1}\right) \mathrm{bh} \mathrm{h}^{\frac{2 \mathrm{n}+1}{\mathrm{n}}}} \mathrm{u}(\varphi)^{\mathrm{a}}\right.$

One can calculate $\eta^{[1])}$ for a first order ODE like equation (4.27) as following [19]:

$\eta_{\varphi}+\left(\eta_{\mathrm{u}}-\xi_{\varphi}\right) \omega-\xi_{\mathrm{u}} \omega^{2}=\xi \omega_{\varphi}+\eta \omega_{\mathrm{u}}$

where $\omega$ is right hand side of (4.27):

$\omega=\left(\frac{V \cos (\varphi)+H \sin (\varphi)}{E\left(\frac{1}{2}\right)^{\frac{n+1}{n}}\left(\frac{n}{2 n+1}\right) b h^{\frac{2 n+1}{n}}}\right) u(\varphi)^{2}$

Substituting $\omega$ and its derivatives in equation (4.28) yields: 


$$
\begin{aligned}
& \eta_{\varphi}+\frac{1}{E\left(\frac{1}{2}\right)^{\frac{n+1}{n}}\left(\frac{n}{2 n+1}\right) b h^{\frac{2 n+1}{n}}} \times\left(\eta_{u}-\xi_{\varphi}\right) \times(V \cos (\varphi)+H \sin (\varphi)) \\
& \times u^{3}\left(\frac{1}{E\left(\frac{1}{2}\right)^{\frac{n+1}{n}}\left(\frac{n}{2 n+1}\right) b h^{\frac{2 n+1}{n}}}\right)^{2} \\
& \times \xi_{\mathrm{u}}(\mathrm{V} \cos (\varphi)+\mathrm{H} \sin (\varphi))^{2} \times \mathrm{u}^{6} \\
& =\frac{1}{E\left(\frac{1}{2}\right)^{\frac{n+1}{n}}\left(\frac{n}{2 n+1}\right) b h^{\frac{2 n+1}{n}}} \times \xi \\
& \times(-V \cos (\varphi)+H \sin (\varphi)) \times u^{3} \\
& +\frac{30}{E\left(\frac{1}{2}\right)^{\frac{n+1}{n}}\left(\frac{n}{2 n+1}\right) b h^{\frac{2 n+1}{n}}} \times \eta \\
& \times(\operatorname{Vcos}(\varphi)+H \sin (\varphi)) \times u^{2}
\end{aligned}
$$

One can show from the above equation:

$$
\eta_{\varphi}=\xi_{\mathrm{u}}=0
$$

Comparison of the coefficients of $\mathrm{u}^{3}$ in equation (4.29) will yield the equation:

$$
\left(\eta_{\mathrm{u}}-\xi_{\varphi}\right)(V \cos (\varphi)+H \sin (\varphi))=\xi(-V \cos (\varphi)+H \sin (\varphi))
$$

To satisfy equation (4.29), $\xi$ must be assumed to be zero otherwise there is no other acceptable value for it.

$$
\xi=0
$$

hence equation (4.29) will be:

The integral of this ODE is: $\eta=\mathrm{u}^{3}$

So the point symmetry becomes:

$\mathrm{X}=\mathrm{u}^{\mathrm{a}} \frac{\partial}{\partial \mathrm{u}}$

Based on (4.35), canonical coordinates can be calculated from (4.19) and (4.20) as:

$\mathrm{r}(\varphi, \mathrm{u})=\varphi$

$$
C_{1}=\left(\frac{M_{0}^{n}}{E\left(\frac{1}{2}\right)^{\frac{n+1}{n}}\left(\frac{n}{2 n+1}\right) b h^{\frac{2 n+1}{n}}}\right)^{2}-\left(\frac{2}{E\left(\frac{1}{2}\right)^{\frac{n+1}{n}}\left(\frac{n}{2 n+1}\right) b h^{\frac{2 n+1}{n}}}\right) \times\left(-V \cos \left(\theta_{f}\right)+H \sin \left(\theta_{f}\right)\right)
$$

where $\theta_{\mathrm{f}}$ is final angle in the tip. Solution of (4.40) yields:

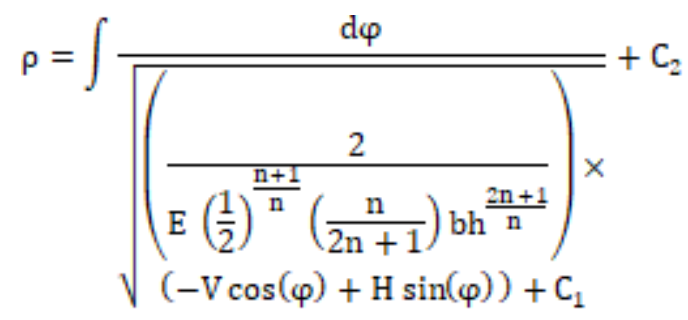

From equations (4.3) and (4.4) one can dive the following equation :

$$
\begin{aligned}
& s(\varphi, u)=\frac{1}{2 u^{2}} \\
& \frac{d s}{d r}=\left(\frac{V \cos (r)+H \sin (r)}{E\left(\frac{1}{2}\right)^{\frac{n+1}{n}}\left(\frac{n}{2 n+1}\right) b h^{\frac{2 n+1}{n}}}\right) \\
& S=\left(\frac{V \cos (r)+H \sin (r)}{E\left(\frac{1}{2}\right)^{\frac{n+1}{n}}\left(\frac{n}{2 n+1}\right) b h^{\frac{2 n+1}{n}}}\right)+C_{1}
\end{aligned}
$$

Further, by substituting (4.36), (4.37) and (4.39) in (4.27) one can get:

$$
\left(\frac{\mathrm{d} \varphi}{\mathrm{d} \rho}\right)^{2}=\left(\frac{2}{\mathrm{E}\left(\frac{1}{2}\right)^{\frac{\mathrm{n}+1}{\mathrm{n}}}\left(\frac{\mathrm{n}}{2 \mathrm{n}+1}\right) \mathrm{bh} \frac{2 \mathrm{n}+1}{\mathrm{n}}}\right) \times(-V \cos (\mathrm{r})+\mathrm{H} \sin (\mathrm{r}))+\mathrm{C}_{1}
$$

$\mathrm{C}_{1}$ can be calculated as following by considering boundary condition (4.6):

$$
\begin{aligned}
& \int_{0}^{a} d x=\int_{0}^{B_{f}} \frac{d \varphi \cos (\varphi)}{\left.\sqrt{\left(\frac{1}{2}\right)^{\frac{n+1}{n}}\left(\frac{n}{2 n+1}\right) b h^{\frac{2 n+1}{n}}}\right) x} \\
& \sqrt{(-V \cos (\varphi)+H \sin (\varphi))+C_{1}} \\
& \int_{0}^{b} d y=\int_{0}^{\theta_{0}} \\
& \theta_{f} \quad d \varphi \sin (\varphi) \\
& \sqrt{\left(\frac{2}{E\left(\frac{1}{2}\right)^{\frac{n+1}{n}}\left(\frac{n}{2 n+1}\right) b h^{\frac{2 n+1}{n}}}\right) x}
\end{aligned}
$$


Which is the relationship that after the integration will yield the deflection at the tip of the not-straight cantilever beam.

\section{Validation}

To verify the solution of equations (4.43) and (4.44), a simplified case of loading that has already been presented in the open literature [5] is analysed based on the above derived solution. The validation requires considering same conditions of loading of same type of beam that it would perform of same fashion.

The assumed beam is straight as the solution in [5] is for a straight beam. In the equations the beams are described by the length (L), width (b) and thickness

(h) - the geometry and the elastic modulus constant (E). The tip deflection for the two models is compared. The analytical formulation of the proposed model coincides with the solution presented in [5].

If one assumes there is no vertical and horizontal forces and there is one constant moment $\mathrm{M}_{0}$.

Equations (4.42), (4.43) and (4.44) will be simplified as following, respectively:

$$
C_{1}=\left(\frac{M_{0}^{n}}{E\left(\frac{1}{2}\right)^{\frac{n+1}{n}}\left(\frac{n}{2 n+1}\right) b h^{\frac{2 n+1}{n}}}\right)^{2}
$$

$$
\begin{aligned}
& \delta_{\mathrm{h}}=\int_{0}^{a} d x=\int_{0}^{\theta_{\mathrm{f}}} \frac{d \varphi \cos (\varphi)}{\sqrt{C_{1}}} \\
& =\int_{0}^{\theta_{f}} \frac{E\left(\frac{1}{2}\right)^{\frac{n+1}{n}}\left(\frac{n}{2 n+1}\right) b h \frac{2 n+1}{n}}{M_{0}^{n}} \cos (\varphi) d \varphi \\
& =L-\frac{E\left(\frac{1}{2}\right)^{\frac{n+1}{n}}\left(\frac{n}{2 n+1}\right) b h^{\frac{2 n+1}{n}}}{M_{0}^{n}} \times \sin \left(\frac{M_{0}^{n}}{E\left(\frac{1}{2}\right)^{\frac{n+1}{n}}\left(\frac{n}{2 n+1}\right) b h^{\frac{2 n+1}{n}}} L\right) \\
& \delta_{v}=\int_{0}^{b} d y=\int_{0}^{\theta_{f}} \frac{d \varphi \sin (\varphi)}{\sqrt{C_{1}}} \\
& =\int_{0}^{\theta_{\mathrm{f}}} \frac{E\left(\frac{1}{2}\right)^{\frac{\mathrm{n}+1}{\mathrm{n}}}\left(\frac{\mathrm{n}}{2 \mathrm{n}+1}\right) \mathbf{b h}^{\frac{2 \mathrm{n}+1}{\mathrm{n}}}}{\mathrm{M}_{0}^{\mathrm{n}}} \times \sin (\varphi) \mathrm{d} \varphi=\frac{\mathrm{E}\left(\frac{1}{2}\right)^{\frac{\mathrm{n}+1}{\mathrm{n}}}\left(\frac{\mathrm{n}}{2 \mathrm{n}+1}\right) \mathbf{b h} \mathbf{h}^{\frac{2 \mathrm{n}+1}{\mathrm{n}}}}{\mathrm{M}_{0}^{\mathrm{n}}} \times(1 \\
& -\cos \left(\frac{M_{0}^{n}}{E\left(\frac{1}{2}\right)^{\frac{n+1}{n}}\left(\frac{n}{2 n+1}\right) b^{\frac{2 n+1}{n}}} L\right)
\end{aligned}
$$

These results as shown in (4.44) and (4.45) coincide with the ones provided in reference [4]. The derived solution represents a general case of the particular solution available in the open literature. The general result could be used for applications of AFMs made from nano-composites that may exhibit some curvature while the material may not have a linear behavior. However, the applications for the proposed solution are not limited to AFM). The analytical solution comes handy in the design phase.

\section{Conclusion}

The deflection of geometrically non-straight cantilever beams subjected point loads and made from materials behaving non-linearly such as the micro-beams used in AFM application was found analytically using Lie symmetry method which enable the reduction of the ODE describing the large deflection of the beam. Such a solution is available in the open literature for only a particular case whereas the proposed method provides a general solution for more complex geometries and loadings. Hereby general analytical solution of the deflection for the not-straight beam was methodically presented. Lie group symmetry method is a universal and powerful method for solving nonlinear differential equations.

\section{References:}

[1] M. Brojan, T. Videnic, a.o. Large deflections of nonlinearly elastic non-prismatic cantilever beams made from materials obeying the generalized Ludwick constitutive law, Meccanica, 2009. 44:733- 739

[2] Bisshopp, K. E., and D. C. Drucker. Large deflection of cantilever beams. Quarterly of Applied Mathematics 3.3 (1945): 272-275.

[3] Wang, T.M., S.L. Lee, and O.C. Zienkiewicz, A numerical analysis of large deflections of beams, International Journal of Mechanical Sciences, 1961.3(3):p.219-228.

[4] Kemper, J.D., Large deflections of tapered cantilever beams. International Journal of Mechanical Sciences, 1968. 10(6): p. 469-478.

[5] Lewis, G. and F. Monasa, Large deflections of cantilever beams of non-linear materials of the Ludwick type subjected to an end moment. International Journal of Non-Linear Mechanics, 1982. 17(1): p. 1-6. 
[6] Monasa, F. and G. Lewis, Large deflections of point loaded cantilevers with nonlinear behaviour. Zeitschrift für Angewandte Mathematik und Physik (ZAMP), 1983. 34(1): p. 124-130.

[7] Ang, Marcel H., Wang Wei, and Low Teck-Seng, On the estimation of the large deflection of a cantilever beam, Industrial Electronics, Control, and Instrumentation, 1993. Proceedings of the IECON'93., International Conference on. IEEE, 1993

[8] Lee, K., Large deflections of cantilever beams of non-linear elastic material under a combined loading. International Journal of Non-Linear Mechanics, 2002. 37(3): p. 439-443

[9] Beléndez, T., C. Neipp, and A. Beléndez, Large and small deflections of a cantilever beam. European journal of physics, 2002. 23: p. 371.

[10] Dado, M. and S. Al-Sadder, A new technique for large deflection analysis of non-prismatic cantilever beams. Mechanics Research Communications, 2005. 32(6): p. 692-703.

[11] Baykara, C., U. Guven, and I. Bayer, Large deflections of a cantilever beam of nonlinear bimodulus material subjected to an end moment. Journal of Reinforced Plastics and Composites, 2005. 24(12): p. 1321-1326.

[12] Belendez, T., et al., Numerical and experimental analysis of large deflections of cantilever beams under a combined load. Physica scripta, 2005. 2005: p. 61.

[13] Al-Sadder, S. and R.A.O. Al-Rawi, Finite difference scheme for large-deflection analysis of non-prismatic cantilever beams subjected to different types of continuous and discontinuous loadings. Archive of Applied Mechanics, 2006. 75(8): p. 459-473.

[14] Wang, J., J.K. Chen, and S. Liao, An explicit solution of the large deformation of a cantilever beam under point load at the free tip. Journal of Computational and Applied Mathematics, 2008. 212(2): p. 320-330.

[15] Shvartsman, B.S., Large deflections of a cantilever beam subjected to a follower force. Journal of Sound and Vibration, 2007. 304(3-5): p. 969-973.

[16] Changizi, Stiharu, Sensitivity Analysis of the Non- Linear Deflection of Non-Straight AFM Micro- Cantilever, Journal of Advanced Microscopy Research, Volume 7, Number 1, March 2012, pp. 51-63(13)

[17] Athisakul, Chainarong, et al. Effect of material nonlinearity on large deflection of variable-arc- length beams subjected to uniform self- weight, Mathematical Problems in Engineering (2012). DOI:10.1155/2012/345461

[18] Changizi, Stiharu, Sahin, A new Approach for the non-linear Analysis of the Deflection of Beams Using Lie Symmetry Groups, Proceedings of the International Conference of Mechatronics and Cyber-MixMechatronics, 2017, Sep. 9-11, Bucharest, Romania, doi.org/10.1007/978-3319-63091-5_17

[19] Changizi, Geometry and Material Nonlinearity Effects on Static and Dynamics Performance of MEMS, PhD thesis, Concordia University, Canada, 2011 